\title{
Metabolomic insights into the intricate gut microbial-host interaction in the development of obesity and type 2 diabetes
}

\section{OPEN ACCESS}

Edited by:

Nuria Salazar,

Instituto de Productos Lácteos de Asturias - Consejo Superior de Investigaciones Científicas, Spain

Reviewed by:

Borja Sanchez,

Instituto de Productos Lácteos

de Asturias - Consejo Superior de Investigaciones Cientificas, Spain

Daniel Monleon Salvado

INCLIVA Research Institute, Spain Maria Victoria Selma Consejo Superior de Investigaciones Cientificas, Spain

${ }^{*}$ Correspondence: Sara Tulipani sara.tulipani@ub.edu; Cristina Andres-Lacueva candres@ub.edu

tThese authors have contributed equally to this work.

Specialty section

This article was submitted to Microbial Physiology and Metabolism, a section of the journal Frontiers in Microbiology

Received: 03 June 2015 Accepted: 05 October 2015 Published: 27 October 2015

Citation:

Palau-Rodriguez M, Tulipani S, Queipo-Ortuño MI, Urpi-Sarda M, Tinahones FJ and Andres-Lacueva C

(2015) Metabolomic insights into the intricate gut microbial-host interaction in the development of obesity and type 2 diabetes.

Front. Microbiol. 6:1151. doi: 10.3389/fmicb.2015.01151
Magali Palau-Rodriguez ${ }^{1 \dagger}$, Sara Tulipani ${ }^{1,2 *}$, Maria Isabel Queipo-Ortuño $^{2,3}$,
Mireia Urpi-Sarda1

${ }^{1}$ Biomarkers and Nutrimetabolomic Lab., Nutrition and Food Science Department, XaRTA, INSA, Campus Torribera, Pharmacy Faculty, University of Barcelona, Barcelona, Spain, ${ }^{2}$ Biomedical Research Institute (IBIMA), Service of Endocrinology and Nutrition, Malaga Hospital Complex Nirgen de la Victoria), University of Malaga, Malaga, Spain, ${ }^{3}$ CIBER Fisiopatología de la Obesidad y Nutrición (CIBERobn), Instituto de Salud Carlos III (ISCIII), Madrid, Spain

Gut microbiota has recently been proposed as a crucial environmental factor in the development of metabolic diseases such as obesity and type 2 diabetes, mainly due to its contribution in the modulation of several processes including host energy metabolism, gut epithelial permeability, gut peptide hormone secretion, and host inflammatory state. Since the symbiotic interaction between the gut microbiota and the host is essentially reflected in specific metabolic signatures, much expectation is placed on the application of metabolomic approaches to unveil the key mechanisms linking the gut microbiota composition and activity with disease development. The present review aims to summarize the gut microbial-host co-metabolites identified so far by targeted and untargeted metabolomic studies in humans, in association with impaired glucose homeostasis and/or obesity. An alteration of the co-metabolism of bile acids, branched fatty acids, choline, vitamins (i.e., niacin), purines, and phenolic compounds has been associated so far with the obese or diabese phenotype, in respect to healthy controls. Furthermore, anti-diabetic treatments such as metformin and sulfonylurea have been observed to modulate the gut microbiota or at least their metabolic profiles, thereby potentially affecting insulin resistance through indirect mechanisms still unknown. Despite the scarcity of the metabolomic studies currently available on the microbial-host crosstalk, the data-driven results largely confirmed findings independently obtained from in vitro and animal model studies, putting forward the mechanisms underlying the implication of a dysfunctional gut microbiota in the development of metabolic disorders.

Keywords: metabolomics, gut microbiota, obesity, type 2 diabetes, co-metabolism

Abbreviations: ${ }^{1} \mathrm{H}-\mathrm{NMR}$, proton nuclear magnetic resonance; BA, bile acids; IGT, impaired glucose tolerance; FMO3, flavin monooxygenase 3; FXR, Farnesoid X Receptor; GC, gas chromatography; LC, liquid chromatography; MS, mass spectrometry; NAFLD, non-alcoholic fatty liver disease; OGTT, oral glucose tolerance test; T2D, type 2 diabetes; TGR-5, $G$-protein coupled receptor; TMA, trimethylamine; TMAO, trimethylamine $N$-oxide. 


\section{GUT MICROBIOTA AND DIABESITY: ROLE IN ENERGY HARVEST, GUT BARRIER INTEGRITY, ENDOCRINE MODULATION, AND METABOLIC INFLAMMATION}

Obesity is a complex,multifactorial disease characterized by an excessive accumulation of fat due to an imbalance between energy intake and expenditure. The linear rise in the prevalence of T2D throughout the normal, overweight and obese ranges is so high that the relative risks of diabetes are 40 times higher when BMI increases above $35 \mathrm{~kg} / \mathrm{m}^{2}$ (Hu et al., 2001; Mokdad et al., 2003; Poirier et al., 2006; World Health Organization [WHO], 2013). The public concern over the obesity epidemic mostly lies in the intimate connection between obesity and T2D (so-called diabesity; Astrup and Finer, 2000) and makes the elucidation of mechanisms underlying the co-occurrence of the two diseases a central focus of current biomedical research.

Recently, consideration has started to be given to the gastrointestinal tract as a key point in the development and progression of complex metabolic diseases, since it represents the milieu where interactions between exogenous (i.e., diet, microbiome) and endogenous (i.e., genetic) factors predisposed to disease and the body's defenses (physical barrier, immune system response) actually take place. Increasing evidence indicates in particular the impact of changes in the composition of the human gut microbiota on host metabolism and a variety of diseases (Bäckhed et al., 2005; Moreno-Indias et al., 2014; Shoaie et al., 2015).

Firmicutes (Gram-positive), Bacteroidetes (Gram-negative) and Actinobacteria (Gram-positive) represent over $90 \%$ of the phyla and dominate the gut microbiota (DiBaise et al., 2008), but a relevant change in their relative proportion has been described in obesity and T2D. A favorable prevalence of Firmicutes bacteria toward healthy subjects has been observed in both animal models of obesity (Ley et al., 2005) and human obesity (Ley et al., 2006; Turnbaugh and Gordon, 2009), also reviewed in (Turnbaugh and Gordon, 2009; Sanz et al., 2013; Moreno-Indias et al., 2014), although with some discrepancies among data (Schwiertz et al., 2010). Although the potential impact of specific species on host metabolism has already been elucidated, most of the data so far available have reported observed changes at the phylum level. Furthermore, the physiological contribution of Firmicutes in the development of the obese phenotype is still being debated. In turn, some studies have observed a positive correlation between ratios of Bacteroidetes to Firmicutes and plasma glucose concentration, but not with BMI, although this was expected (Larsen et al., 2010).

Different mechanisms have been proposed in the attempt to understand the impact of microbiota both in maintaining metabolic health and in the development of obesity and T2D. Essentially, the intestinal microbial variability has been hypothesized as an important factor in four different processes, namely: (i) the modulation of energy homeostasis by regulating the energy harvest from diet, fat storage, lipogenesis, and fatty acid oxidation (host energy metabolism; Tilg et al., 2009; Musso et al., 2010); (ii) the modulation of the gut barrier integrity by regulating the epithelial permeability, the intestinal motility and the transport of digestion products such as short-chain fatty acids, which are an energy source for colonocytes (Samuel et al., 2008); (iii) the regulation of gastrointestinal peptide hormone secretion, by suppressing the secretion of the lipoprotein lipase inhibitor (fasting-induced adipose factor), determining the release of fatty acids from circulating triglycerides and lipoproteins in muscle and adipose tissue and promoting fat mass accumulation (Bäckhed et al., 2007); and (iv) the modulation of the host inflammatory state by contributing to the systemic increase of lipopolysaccharide, which impairs insulin sensitivity (metabolic endotoxemia; reviewed in Bäckhed et al., 2007; Cani et al., 2007, 2012; Sun et al., 2010; Vrieze et al., 2010; Shen et al., 2013). Evidence of the role of gut microbiota in the preservation of metabolic health also comes from the effect of prebiotics, such as non-digestible carbohydrates, namely non-digestible ingredients that are fermented by specific beneficial bacterial strains, selectively promote the growth and/or activity (release of end-products of bacterial fermentation) of the gastrointestinal microbiota, affecting favorably the host health (Gibson et al., 2010). The intake of prebiotics has in fact been described to act on host endocrine secretion, improve gut barrier integrity by increasing the release of glucagon-like peptide-2 (Cani et al., 2012; Dewulf et al., 2013), stimulate postprandial release of peptides involved in energy homeostasis and/or pancreatic functions such as the anorexigenic glucagon-like peptide-1 and peptide YY, and the decrease of orexigenic peptides such as ghrelin in plasma which in turn modulates food intake (regulators of appetite) and energy expenditure across the entire gastrointestinal tract (Piche et al., 2003; Delzenne and Cani, 2011; reviewed in Vrieze et al., 2010). Furthermore, evidence suggests that the modulation of the host metabolic health by prebiotics intake can be mediated to specific fermentation products (i.e., short-chain fatty acids, predominantly acetate, propionate and butyrate) produced by cross-feeding between Eubacterium rectale and Bifidobacterium thetaiotaomicron (Venema, 2010); Propionibacterium sp. and Bacteroides sp. (Hosseini et al., 2011); Faecalibacterium prausnitzii and Roseburia intestinalis/Eubacterium rectale (Duncan et al., 2004; Venema, 2010) respectively.

\section{THE METABOLOMIC APPROACH}

Due to the species specificity of several enzymatic machineries, the gut microbial composition and activity are likely to be characterized by the profile of small metabolites produced in the intestinal lumen, eventually absorbed through the intestinal barrier and further biotransformed by the host. Consequently, the complexity of microbial-host exchanges may be reflected in the specific chemical signature of host circulating biofluids (Nicholson et al., 2012). Metabolomics has recently attracted attention as the most suitable -omics technology for investigating complex, polygenic and multifactorial diseases with a strong metabolic etiology, such as obesity and T2D as well as the crosstalk of distinct predisposing factors in disease development 
and progression (Faber et al., 2007; Llorach et al., 2012; Du et al., 2013; Kurland et al., 2013). Aimed at the comprehensive analysis of the low- molecular- weight compounds contained in a biological system -by definition, metabolites comprise a plethora of primary or secondary derivatives of the intermediate metabolism (molecular weight below 900 and 2000 Dalton, depending on sources; Beckonert et al., 2010; Psychogios et al., 2011; Hadacek, 2015) metabolomics represents a powerful tool for exploring the crosstalk between the microbial and host metabolism in a more exhaustive fashion.

The workflow applied in metabolomic studies is broadly categorized into five main steps: (1) sample collection, (2) sample preparation, (3) data acquisition, (4) data analysis, and (5) biological interpretation of the results obtained (Llorach et al., 2012). The analytical techniques most commonly used for the characterization of the metabolome of a biological sample are MS and ${ }^{1} \mathrm{H}$-NMR. Both technologies have their advantages and disadvantages. ${ }^{1} \mathrm{H}$-NMR implies a non-destructive, non-selective, cost-effective, and relatively sensitive analysis while, compared to ${ }^{1} \mathrm{H}$-NMR, MS mainly offers potential advantages in terms of sensitivity and, if coupled to different separation techniques such as LC or GC, it provides a means of detecting a broader and complementary range of biomarkers (Faber et al., 2007). LC coupled to electrospray ionization MS is becoming the method of choice for the acquisition of profiling metabolites in complex biological samples (Scalbert et al., 2009) through both targeted (i.e., triple quadrupole-driven) and non-targeted (e.g., quadrupole time-of-flight-, linear trap quadrupole orbitrapdriven) approaches.

The present review aims to summarize the gut microbialhost cometabolites identified so far in humans in relation to obesity and/or T2D by targeted and untargeted metabolomic studies. Since the potential impact of some specific species in host metabolism has already been elucidated, an attempt to associate bacterial producers of the co-metabolites with the metabolic alterations related to the obese, diabetic, or diabese phenotype was also made. A critical view of the current limitations and future directions of metabolomics will accompany the discussion.

\section{MATERIALS AND METHODS}

\section{Search Strategy}

The following keywords were searched for in the PubMed and Web of Science electronic databases: (Metabolom* [TW] or co-metabol* [TW] or host-gut metabo* [TW] or nuclear magnetic resonance [TW] or MS [TW] or magnetic resonance spectroscopy [TW]) AND (OBES* [TW] OR DIABET* [TW] OR DIABES* [TW]) AND (gut micro* [TW]). Species (human), language (English), and publication date restrictions (2000 to date, last search on November 27th, 2014) were imposed, but there were none for gender, age or ethnicity. Relevant references cited in the selected articles were additionally reviewed. Targeted and untargeted metabolomic approaches driven by ${ }^{1} \mathrm{H}-\mathrm{NMR}$ or MS techniques were both included in the selection. Lowmolecular-weight $(<1000 \mathrm{Da})$ metabolites significantly up- or downregulated in overweight and obese subjects with/without impaired glycemic control, with respect to controls (i.e., lean, healthy subjects), were the primary outcomes of interest of the review.

\section{RESULTS AND DISCUSSION}

\section{Characteristics of the Studies and Metabolic Variations}

Only eight human studies successfully met the eligibility criteria for inclusion in the review (details in the Supplementary Material File). As summarized in Table 1, seven observational and one interventional study have so far applied a metabolomic approach and specifically identified changes in products of the gut microbial-host co-metabolism in overweight to obese individuals $\left(\mathrm{BMI}>25 \mathrm{~kg} / \mathrm{m}^{2}\right)$ and/or several degrees of impaired glycemic control (ranging from IGT up to T2D) compared to control individuals. Other comorbidities were not described (i.e., hypertension, renal or liver dysfunction).

Overall, the study subjects, designs and objectives were quite heterogeneous despite the small number of retrieved studies (Supplementary Material File), thereby complicating an otherwise integrated and consistent picture of the metabolomic changes observed.

Urine (Salek et al., 2007; Calvani et al., 2010; Zhao et al., 2010; Huo et al., 2015), fasting serum (Huo et al., 2009; Zhang et al., 2009; Suhre et al., 2010) and plasma (Zhao et al., 2010; Campbell et al., 2014) were the biological samples used in these studies. A data-driven untargeted approach was chosen in the majority of the studies (Salek et al., 2007; Huo et al., 2009; Zhang et al., 2009; Calvani et al., 2010; Zhao et al., 2010; Campbell et al., 2014) while two of them provided quantitative information about known targeted metabolites (Suhre et al., 2010; Huo et al., 2015). The metabolic changes observed in these studies and the related interpretations are summarized in Table 2.

\section{Co-metabolism of Bile Acids}

Two of the metabolomic studies described in this review highlighted a change in the circulating pool of BA in obese patients with insulin resistance or T2D, compared with BMImatched healthy individuals (Suhre et al., 2010; Zhao et al., 2010). Alterations involved both human-derived (hepatic) structures (primary BA) and gut microbial-produced derivatives (secondary BA).

To the best of our knowledge, it is currently accepted that the bacterial enzymes involved in the biotransformation from primary to secondary BA are not shared across the whole microbial community, although they have been described so far in genera belonging to the four major phyla Firmicutes, Actinobacteria, Bacteroidetes and Proteobacteria (Labbé et al., 2014). Furthermore, according to Jones et al. (2014) Actinobacteria and Firmicutes clones would be the only ones able to degrade all conjugated BA, with Bacteroidetes species being limited to tauro- conjugation activities.

After their production in the liver and the eventual glycoand tauro-conjugation $(N$-acyl amidation with glycine or taurine 


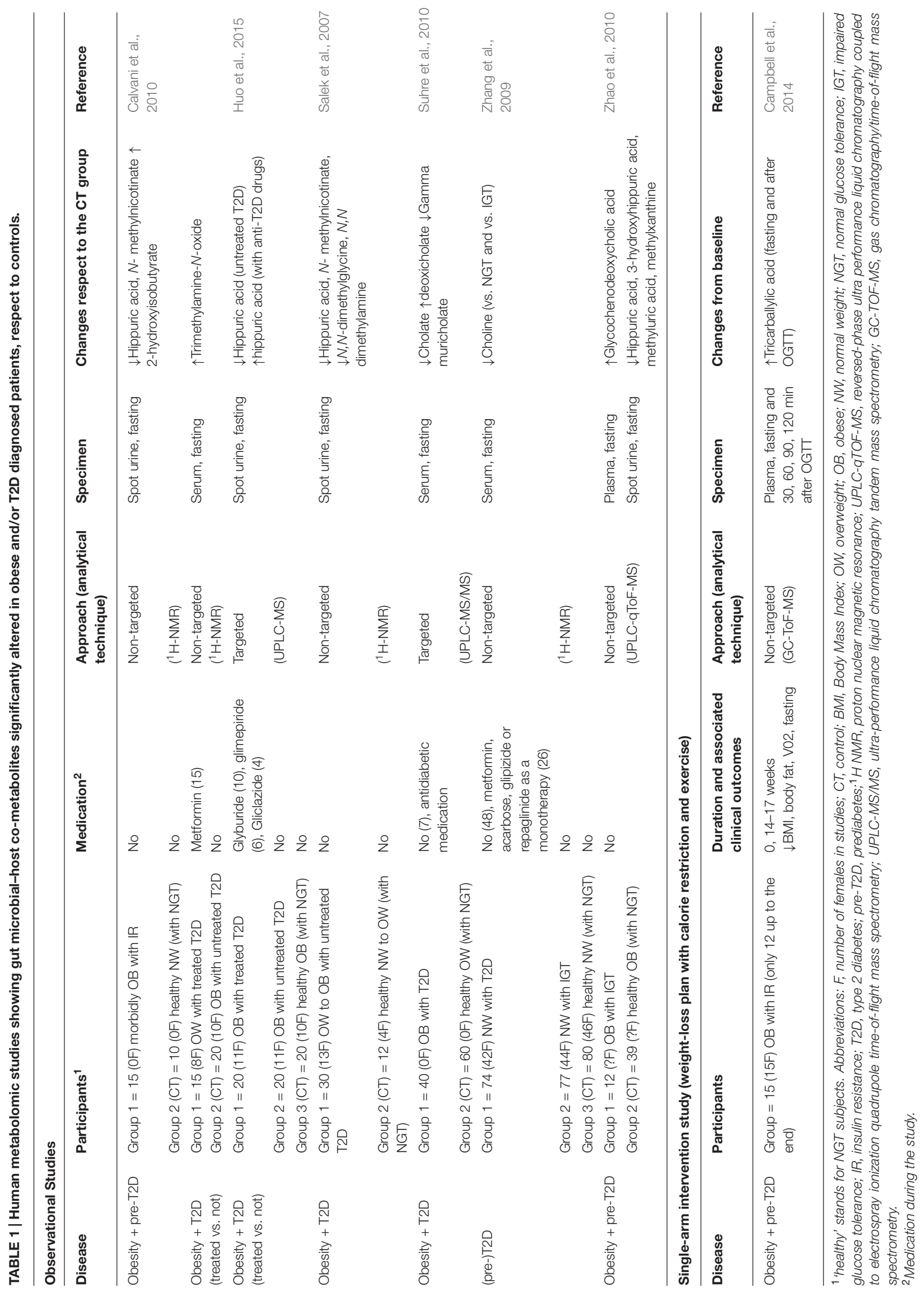




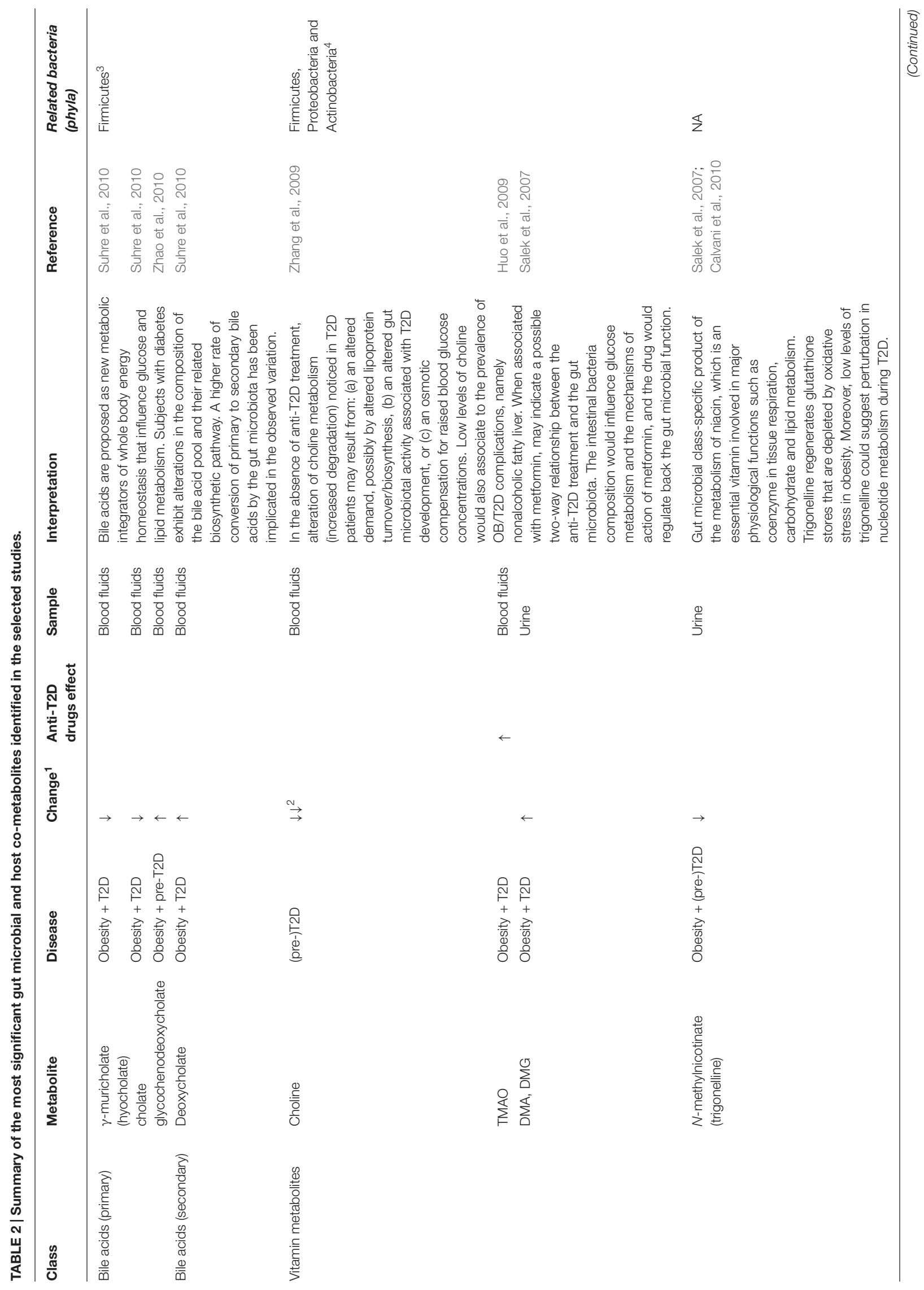




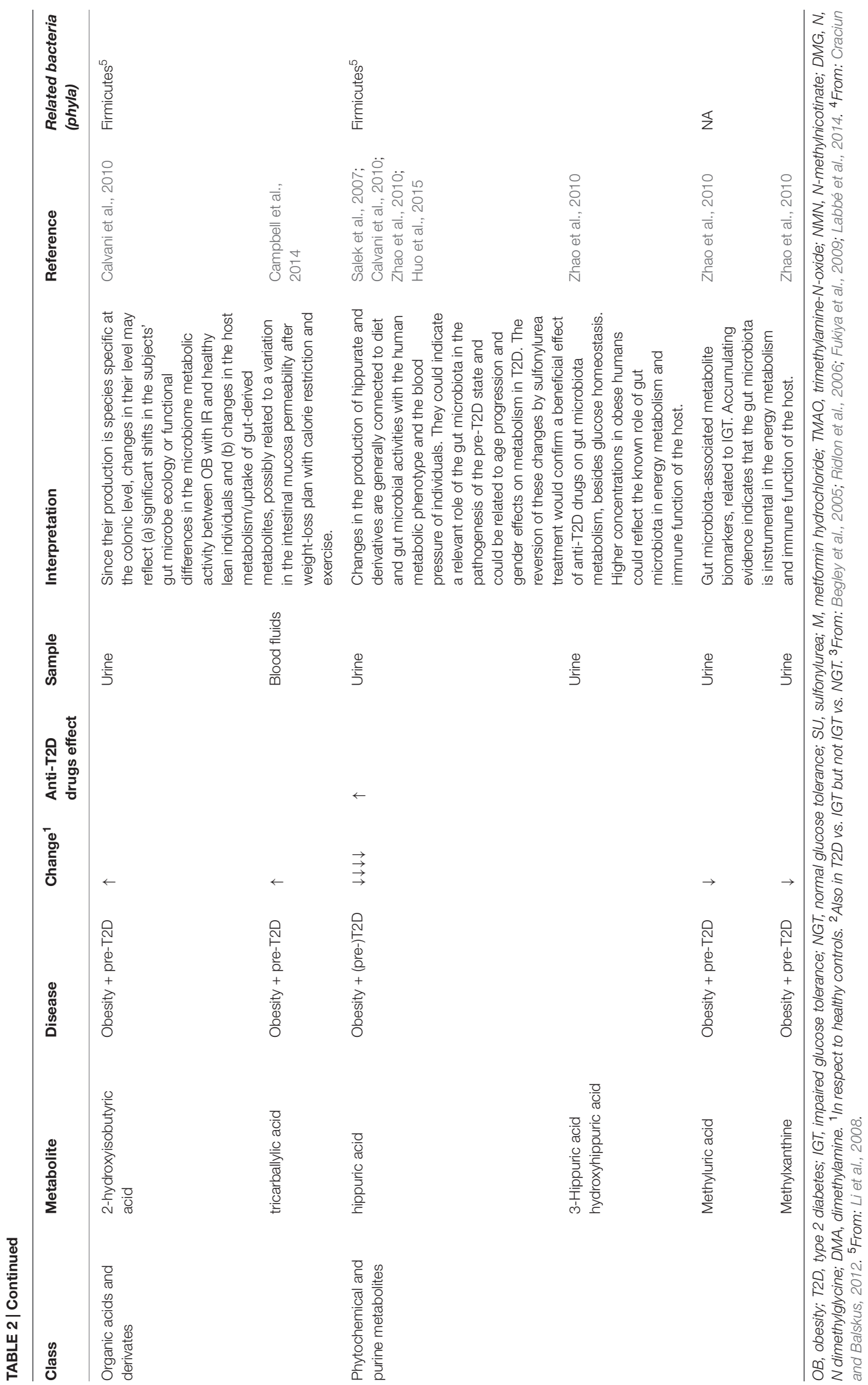


substituents), primary BA are secreted in the small intestine through the bilis (Ridlon et al., 2006; Hofmann and Hagey, 2008; Swann et al., 2011), where they are first subjected to deconjugation by a bacterial bile salt hydrolase enzyme produced by species of the four phyla, such as Clostridium, Bacteroides, Lactobacillus, Bifidobacterium, and Enterococcus (Begley et al., 2005). In the ileum, more than $95 \%$ of these BA undergo enterohepatic recycling (Swann et al., 2011; Kootte et al., 2012; Nicholson et al., 2012), are absorbed from the intestine and returned to the liver (Ridlon et al., 2006). The remaining $5 \%$ escape the enterohepatic circulation and reach the large bowel where the bioconversion into secondary BA is completed, especially by Firmicutes phyla (Eubacterium sp. and Clostridium sp.; Midtvedt, 1974; Nguyen and Bouscarel, 2008; Swann et al., 2011).

A decrease of primary BA and an increase of secondary BA was observed in the fasting serum of overweight patients with T2D, compared to healthy subjects (Suhre et al., 2010). The authors hence concluded that differences in the gut microbiota of diabetic patients may lead to higher rates of conversion from primary to secondary BA. In turn, Zhao et al. (2010) only observed an increase of glycochenodeoxycholic acid (primary BA) in the plasma of prediabetic individuals, while no changes were noticed in urine. Despite the only partial overlapping of the results, both studies suggested that overweight and obesity may not be the predominant factor implied in the metabolomic changes observed, and thus in linking impaired glucose homeostasis to alterations in BA pool composition. As argued in those studies, the variation of the BA pool in biofluids may depend on different factors, namely a change in the prevalence or activation of the gut microbial species implied in BA bioconversion or an altered reabsorption of BA through the gut mucosa, in turn produced by the disease itself, by dietary or other external changes (i.e., induced by bariatric surgery), or by a combination of these. In any case, the results indicated a probable implication of the modulation of BA biosynthetic pathways in the relationship between gut microbiota and insulin resistance (Figure 1).

These findings are in line with independent studies that recently associated changes in BA turnover with diabesity. In turn, a reduction of the bacterial enzymatic activities involved in the conversion of primary into secondary BA was observed in diabetic patients compared to healthy controls, and linked to Firmicutes phyla (Labbé et al., 2014). A very similar pattern was also reported in obese patients with diagnosed metabolic syndrome, treated with antibiotic agents (vancomycin and amoxicillin) and associated with both a decreased prevalence of the Firmicutes population and a reduction of peripheral insulin sensitivity (Vrieze et al., 2014). Taken together, these data suggest a possible link between BA and metabolic health (Lefebvre et al., 2009). In line with these findings, BA have recently been proposed as metabolic integrators of whole-body energy homeostasis implicated in the regulation of various metabolic pathways, including their own synthesis and enterohepatic circulation, triglyceride, glucose, and energy homeostasis, by acting as signaling molecules through receptor-dependent and -independent pathways. The role of a dysregulation of this

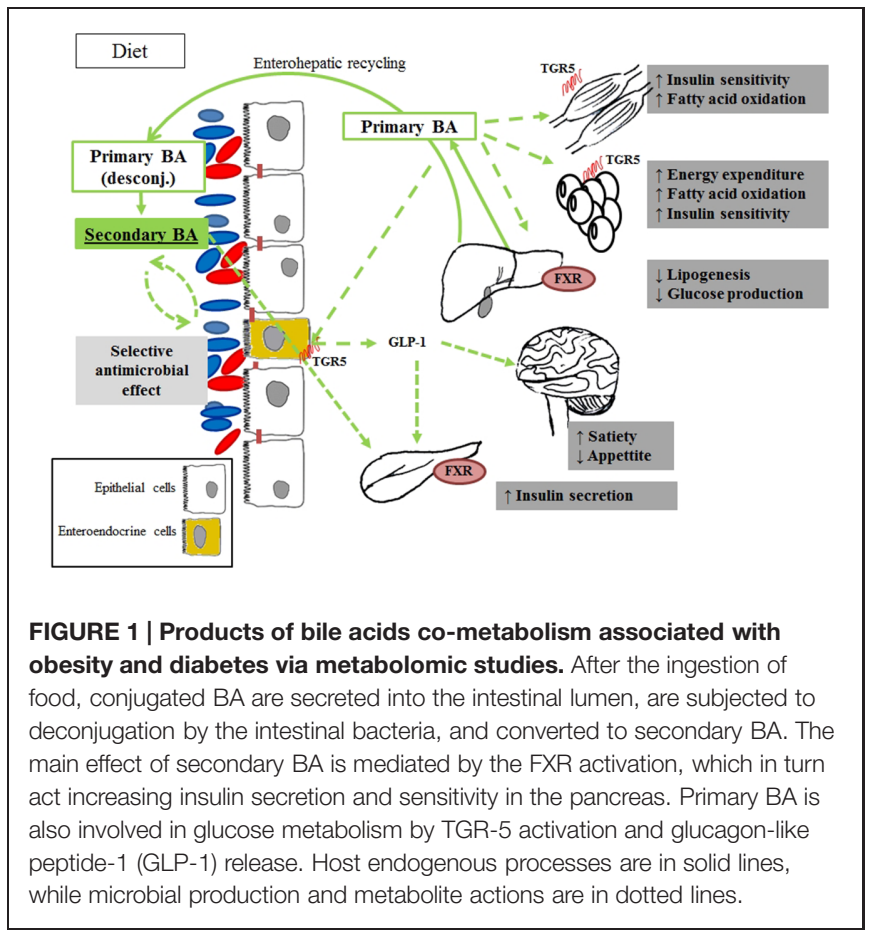

BA-mediated metabolic control in the pathogenesis of T2D and co-morbidities, such as its attractiveness as a therapeutic target, is now beginning to be elucidated (reviewed in Prawitt et al., 2011). Their action on energy metabolism regulation would occur via both the activation of the nuclear receptor FXR and FXR-independent pathways, such as through the membrane receptor TGR5 expressed in several tissues including gall bladder, ileum, colon, and brown and white adipose tissue.

It is worth noting that preliminary evidence has shown that not all BA activate equally, and the microbial-derived production of secondary BA could be an important mechanism in the regulation of signaling pathways involved in the development of diabesity (Nguyen and Bouscarel, 2008). When gut microbial-derived secondary BA are bound to TGR5, the receptor is internalized and a series of adenylate cyclasedependent signaling is triggered by activating distinct pathways involved in glucose and lipid energy metabolism (Kawamata et al., 2003; Thomas et al., 2008). In tissues, such as brown adipose tissue and muscle, this would lead to an increased mitochondrial activity and oxidative phosphorylation, which has been linked to an insulin sensitization both in genetic and diet-induced models of diabesity (Watanabe et al., 2006). In enteroendocrine L-cells (Kawamata et al., 2003), in turn, these signaling pathways would enhance glucose metabolism by stimulating the production of glucagon like peptide, thereby promoting insulin secretion. Finally, recent studies have also shown that BA secretion improves insulin secretion, insulin sensitivity and whole-body glucose homeostasis (reviewed in Thomas et al., 2008), improving liver and pancreatic function in obese mice (Thomas et al., 2009; Tremaroli and Bäckhed, 2012). 


\section{Co-metabolism of Vitamins}

Products of the gut microbiota-driven metabolic pathway of vitamins such as choline and niacin have been associated with obesity and diabetes.

\section{Choline Metabolism}

Although humans may produce choline endogenously (de novo hepatic synthesis), dietary intake (e.g., from egg yolk, liver, muscle meats, fish, nuts, legumes) is necessary to meet the demand for body health maintenance (Blusztajn, 1998; Zeisel, 2000). Once dietary choline reaches the intestine, anaerobic intestinal microorganisms, mainly of Firmicutes and Proteobacteria phyla (Romano et al., 2015) catalyze its conversion to TMA, which may be further degraded to several methylamines by the gut microbiota (sym-xenobiotic pathway; Harris et al., 2012), or is absorbed and oxidized to TMAO by the hepatic FMO3 enzyme. Choline may also be converted into betaine and further products (e.g., dimethylglycine) by mammalian mitochondrial pathways in the liver and kidney (Lever and Slow, 2010) (Figure 2). The bacterial gene clusters responsible for anaerobic choline degradation started to be identified only recently. Bacterial species belonging to Firmicutes, Actinobacteria and Proteobacteria phyla have been revealed as possessing the necessary enzymatic activities, while Bacteroidetes would be apparently deprived (Craciun and Balskus, 2012). However, the complete diversity of species that contribute to TMAO production in humans still remains unknown (Romano et al., 2015).

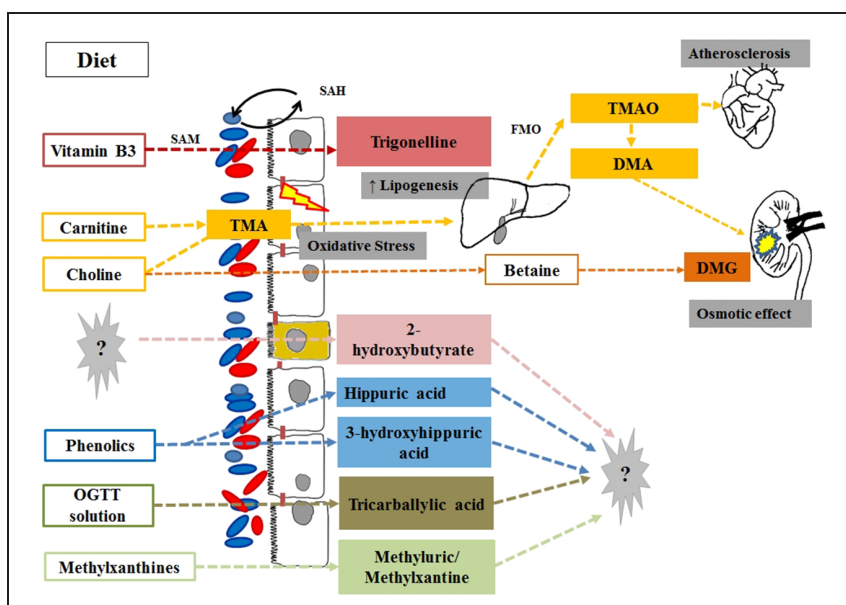

FIGURE 2 | Products of vitamin, phytochemical and purine co-metabolism associated with obesity and diabetes via metabolomics studies. Intestinal microorganisms catalyze the conversion of dietary choline and carnitine into TMA with a direct effect on the intestinal mucose (increased oxidative stress) and is the substrate for the hepatic production of TMAO (associated with cardiovascular disease risk) and TMA. Choline may escape microbial degradation and convert into betaine and further products [i.e., dimethylglycine (DMG)] by mammalian mitochondrial pathways in the liver and kidney where they have an detrimental osmotic effects. For most of the host microbial co-metabolites associated with the diabese phenotype, the eventual role in glucose and lipid metabolism remains unknown. OGTT, Oral glucose tolerance test; SAH and SAM,

$S$-adenosylmethionine to $S$-adenosylhomocysteine (methionine cycle).
Recent studies have shown that circulating levels of choline and TMAO are related to cardiovascular disease risk (Dumas et al., 2006; Micha et al., 2010; Wang et al., 2011; Koeth et al., 2013; Warrier et al., 2015), and the gut microbiota-driven TMAFMO3-TMAO pathway has been particularly recognized as a key regulator of lipid metabolism and inflammation. Increased levels of TMAO have been observed in a leptin-deficient murine model of obesity and T2D (Gipson et al., 2008; Won et al., 2013) and revealed a contribution of gut microbiota to fatty liver phenotype in insulin-resistant mice (Dumas et al., 2006). The systemic perturbations of key metabolites of choline have also been related to the progression of T2D, suggesting that in the early stages of diabetes an attenuated conversion of choline into dimethylglycine may occur, which can be observed by the increased levels of TMAO and TMA, with a reversion of this behavior at a later stage of the disease (Guan et al., 2013).

Messana et al. (1998) published the first study linking TMAO and T2D in humans. Using a ${ }^{1} \mathrm{H}-\mathrm{NMR}$ approach, increased levels of TMAO and dimethylamine were observed in the urine of diabetic individuals compared to a group of healthy individuals, and were present in high concentrations even in diabetics with good metabolic control (i.e., absence of glycosuria and glycohemoglobin). In the last decade, little progress has been made on the mechanisms that would directly or indirectly involve TMAO in the development of diabetes. Nevertheless, the potential role of an altered composition of the microbiota and its ability to metabolize choline in glucose homeostasis and disease development has become increasingly relevant (Dumas et al., 2006).

To the best of our knowledge, three further metabolomic studies observed a change in choline metabolism, which was associated with impaired glycemic control in humans, within a wide range of BMI (Salek et al., 2007; Huo et al., 2009; Zhang et al., 2009; Table 1). In all of them, fasting biosamples were analyzed, thereby avoiding fluctuations in choline concentrations due to dietary intake. Salek et al. (2007) carried out a ${ }^{1}$ H-NMR-driven metabolomic comparison of urinary changes linked to T2D both in animals (obese mice and rats with autosomal recessive defects in the leptin receptor gene $-d b / d b$ and Zucker $f a / f a$, respectively), and individuals who were overweight to obsese $(B M I=25-40)$ compared to healthy lean controls. An increased excretion of a product of choline biotransformation, namely $\mathrm{N}, \mathrm{N}$-dimethylglycine and $\mathrm{N}, \mathrm{N}$-dimethylamine, distinguished the urinary metabolome of T2D in all species in the study (Salek et al., 2007), suggesting a possible increase in the choline turnover. The authors assumed that the diabese phenotype may have a major demand for choline, possibly due to an altered biosynthesis of lipoproteins, an altered metabolism of methylamines - which would play an important osmoregulatory role by degrading dietary choline - or to an altered intestinal microbiota composition (Salek et al., 2007). Although there is a scarcity of data in this regard, Firmicutes and Proteobacteria seem to be the most implicated phyla in the conversion of choline to TMAO (Romano et al., 2015). For this reason, the decline of choline circulating levels and increase of choline subproducts such as TMAO and DMA in obese subjects would be in line 
with the increase of the Firmicutes phylum associated with obese phenotype (Ley et al., 2006).

The role of BMI in the observed association was partly downsized in a second study (Zhang et al., 2009). By applying a similar untargeted and ${ }^{1}$ HNMR-driven approach, in fact, Zhang et al. (2009) demonstrated a decrease in the serum levels of choline in normal-weight subjects with T2D $(\mathrm{BMI}=25.9 \pm 9.0)$ compared to non-diabetic lean individuals (normal or IGT); however, no changes in the downstream products of choline metabolism were detected. Aside from increased insulin resistance, the decrease in serum choline is linked to a specific shift in the gut microbial community in the diabetic patients (relative increase in Firmicutes) and to an increase in the prevalence of T2D complications, as NAFLD (Zhang et al., 2009). The role of the microbial community hosted by diabese subjects in altering choline metabolism was also tested by assessing the effects of antidiabetic medication (Huo et al., 2009). As shown in Table 1, Huo et al. (2015) observed increased serum levels of TMAO in overweight diabetic subjects receiving metformin treatment versus untreated diabese controls, which may indicate an intestinal bacterial regulation function of metformin. It has already been suggested that antidiabetic treatments have a beneficial effect on gut microbiota metabolism (Huo et al., 2015), although the exact mechanisms are still unclear (Moreno-Navarrete et al., 2012). The authors suggested a link between the deregulation of choline metabolism in T2D and a rupture of the intestinal barrier by oxidative stress (Wei et al., 2008). In any case, a possible two-way relationship between anti-T2D treatment and gut microbiota has been hypothesized.

\section{Niacin Metabolism}

Alterations in the niacin (vitamin B3) metabolism have also been observed in association with obesity and T2D, and due to the overlapping in the choline/niacin catabolic pathways (i.e., via betaine and glycine metabolism), may also reflect a dysregulation in choline metabolism (Huang et al., 2012). Through a LC-MS-driven metabolomic approach, an altered urinary excretion of nicotinuric acid ( $N$-nicotinoylglycine) was recently proposed as a potential marker of metabolic syndrome diagnostic traits and of cardiometabolic risk (Huang et al., 2012). Similarly, an association between the presence of trigonelline (betaine nicotinate) and obese and diabetic phenotypes has been proposed. Despite having a possible exogenous (dietary) origin, trigonelline is mostly biosynthesized by the gut microbiota during the conversion of $S$-adenosylmethionine to $S$-adenosylhomocysteine (methionine cycle). By applying a ${ }^{1} \mathrm{H}$-NMR-based metabolomics approach, Salek et al. (2007) found lower levels of trigonelline in the urine of diabetic $(d b / d b)$ mice and humans, associated with a change in energy and tryptophan metabolism. Further animal (Salek et al., 2007; Won et al., 2013) and human studies (Calvani et al., 2010) confirmed a decline of trigonelline in obesity and diabetes, some authors suggesting that oxidative stress possibly has a role (i.e., via glutathione store depletion) in the observed relationship (Calvani et al., 2010). Trigonelline is known to be involved in major physiological functions including lipid and carbohydrate metabolism.

\section{Co-metabolism of Organic Acids and Derivates}

Calvani et al. (2010) identified high levels of 2hydroxyisobutyrate in the urine of obese people, and the change was associated with a reduced bacterial diversity in 'obese' gut microbiota possibly involved in nutrient and energy harvest (Tables 1 and 2). In particular, 2-hydroxyisobutyrate is a product of the microbial degradation of dietary proteins that escape digestion in the upper gastrointestinal tract, and its production has been associated with the presence of specific microbial members such as Faecalibacterium prausnitzii (Li et al., 2008), butyrate-producer species with anti-inflammatory effect and to be in low levels in obese and diabese individuals compared to healthy subjects (Qin et al., 2012). In addition, Campbell et al. (2014) observed that obese subjects involved in a dietary weight loss program had higher levels of tricarballylic acid after an OGTT compared with the fasting concentrations. Interestingly, tricarballylic acid is a product of gut microbial metabolism of food-derived trans-aconitate, described as an additive contained in the OGTT solution. Once again, the authors suggested a two-way relationship between the obese and gut microbial phenotype (tricarballylic acid production would in turn increase the metabolic activity of specific gut microbes associated with its production).

\section{Co-metabolism of Phytochemicals and Purines}

Hippuric acid and 3-hydroxyhippuric acid are two normal urinary components mainly derived from the degradation of plant (poly)phenols and aromatic amino acids (i.e., phenylalanine and tryptophan) by a range of gut microbes, recently found to belong to Clostridium sp. (Li et al., 2008). The resulting benzoic acid is then absorbed, subjected to glycine conjugation reaction (by mitochondrial glycine $\mathrm{N}$-acyltransferase) and finally excreted in urine (Huo et al., 2015). Decreased levels of hippuric acid (Salek et al., 2007; Calvani et al., 2010; Zhao et al., 2010) and 3-hydroxyhippuric acid in urine have been related to IGT and obesity (Zhao et al., 2010) in both animal and human studies. In turn, the downregulation was reduced in T2D patients after the treatment with sulfonylurea, suggesting the drug potentially has a protector effect on gut microbiota metabolism (Huo et al., 2015). However, a strict dietary assessment is mandatory to dismiss any diet-dependent variation among groups, due to the wide range of phenolic compounds leading to these last-step metabolites following microbial and human biotransformations (lack of specificity; Salek et al., 2007). Moreover, the reasons for their putative associations with obesity and T2D are unknown. Salek et al. (2007) suggested that hippurate could be related to age progression and gender effects on metabolism in T2D, but these suppositions need to be further investigated.

Zhao et al. (2010) observed that subjects with IGT had a reduced excretion of methyluric acid and methylxanthine, which are products of the microbial metabolism of methylxanthines contained for instance in coffee and tea. The authors tentatively interpreted the observed changes as the result of an altered gut 
microbiota in the presence of insulin resistance, although their putative role in glucose metabolism is still unknown.

\section{CONCLUSION}

Current public health burdens such as obesity and T2D are complex, polygenic, multifactorial diseases with a strong metabolic etiology. Gut microbiota have recently been proposed as a crucial environmental factor in their development, but the metabolic complexity of the symbiotic interaction between the host individual and its microbial community, as well as the impact of this crosstalk between body weight changes and glucose homeostasis, are still unclear.

However, our review highlighted how few metabolomic studies have been specifically conducted so far to identify the role of the gut microbiota in the development and progression of obesity and T2D, at least in humans.

Despite the scarcity, heterogeneity and intrinsic limitations of the metabolomic studies conducted so far aimed at identifying the role of the gut microbiota in the development and progression of obesity and $\mathrm{T} 2 \mathrm{D}$ (i.e., wide range of BMI and/or glycemic status evaluated, important sources of variability not considered including ethnic, gender effects, and dietary assessments), the results obtained by these data-driven metabolomic approaches are in line with findings independently obtained from in vitro or animal model studies. Products of the microbial/host metabolism of BA, vitamins (choline, niacin), branched fatty acids, purines and phenolic compounds have been described as being altered in (pre-)diabetic subjects, with or without increased BMI, compared with healthy controls. Moreover, few articles show a clear relation between metabolites and their bacterial producers in terms of the complexity

\section{REFERENCES}

Astrup, A., and Finer, N. (2000). Redefining type 2 diabetes: “diabesity" or "obesity dependent diabetes mellitus"? Obes. Rev. 1, 57-59. doi: 10.1046/j.1467789x.2000.00013.x

Bäckhed, F., Ley, R. E., Sonnenburg, J. L., Peterson, D. A., and Gordon, J. I. (2005). Host-bacterial mutualism in the human intestine. Science 307, 1915-1920. doi: 10.1126/science.1104816

Bäckhed, F., Manchester, J. K., Semenkovich, C. F., and Gordon, J. I. (2007). Mechanisms underlying the resistance to diet-induced obesity in germ-free mice. Proc. Natl. Acad. Sci. U.S.A. 104, 979-984. doi: 10.1073/pnas.0605 374104

Beckonert, O., Coen, M., Keun, H. C., Wang, Y., Ebbels, T. M. D., Holmes, E., et al. (2010). High-resolution magic-angle-spinning NMR spectroscopy for metabolic profiling of intact tissues. Nat. Protoc. 5, 1019-1032. doi: 10.1038/nprot.2010.45

Begley, M., Gahan, C. G. M., and Hill, C. (2005). The interaction between bacteria and bile. FEMS Microbiol. Rev. 29, 625-651. doi: 10.1016/j.femsre.2004.09.003

Blusztajn, J. K. (1998). Choline, a vital amine. Science 281, 794-795. doi: 10.1126/science.281.5378.794

Calvani, R., Miccheli, A., Capuani, G., Tomassini Miccheli, A., Puccetti, C., Delfini, M., et al. (2010). Gut microbiome-derived metabolites characterize a peculiar obese urinary metabotype. Int. J. Obes. (Lond.) 34, 1095-1098. doi: 10.1038/ijo.2010.44

Campbell, C., Grapov, D., Fiehn, O., Chandler, C. J., Burnett, D. J., Souza, E. C., et al. (2014). Improved metabolic health alters host metabolism in parallel with of the gut microbiota and the cross-feeding mechanisms that would have combined bacterial effects in the colon ecosystem.

More efforts should be directed in the future toward expanding our knowledge of the metabolic interactions of the host and the gut microbiota, particularly through a strict evaluation of the lifestyle factors (i.e., diet) strongly involved in the modulation of this crosstalk.

\section{ACKNOWLEDGMENTS}

This research was supported by: the PI13/01172 Project (Plan $\mathrm{N}$ de $\mathrm{I}+\mathrm{D}+\mathrm{i}$ 2013-2016) from the Instituto de Salud Carlos III (ISCIII)-Subdirección General de Evaluación y Fomento de la Investigación, and the PI-0557-2013 Project, from Fundación Progreso y Salud Consejería de Salud y Bienestar Social, Junta de Andalucía, both cofounded by the Fondo Europeo de Desarrollo Regional (FEDER); the JPI HDHL FOODBALL (PCIN-2014133-MINECO-Spain), and ISCIII-CIBEROBN. We also thank the award of 2014SGR1566 from the Generalitat de Catalunya's Agency AGAUR. ST acknowledges the Juan de la Cierva fellowship (MINECO). MO acknowledges support from the "Miguel Servet Type I" program (CP13/00065) of the Instituto de Salud Carlos III, Madrid, Spain. MU-S acknowledges the Ramón y Cajal program from MINECO and Fondo Social Europeo.

\section{SUPPLEMENTARY MATERIAL}

The Supplementary Material for this article can be found online at: http://journal.frontiersin.org/article/10.3389/fmicb. 2015.01151

changes in systemic xeno-metabolites of gut origin. PLoS ONE 9:e84260. doi: 10.1371/journal.pone.0084260

Cani, P. D., Amar, J., Iglesias, M. A., Poggi, M., Knauf, C., Bastelica, D., et al. (2007). Metabolic endotoxemia initiates obesity and insulin resistance. Diabetes 56, 1761-1772. doi: 10.2337/db06-1491

Cani, P. D., Osto, M., Geurts, L., and Everard, A. (2012). Involvement of gut microbiota in the development of low-grade inflammation and type 2 diabetes associated with obesity. Gut Microbes 3, 279-288. doi: 10.4161/gmic. 19625

Craciun, S., and Balskus, E. P. (2012). Microbial conversion of choline to trimethylamine requires a glycyl radical enzyme. Proc. Natl. Acad. Sci. U.S.A. 109, 21307-21312. doi: 10.1073/pnas.1215689109

Delzenne, N. M., and Cani, P. D. (2011). Interaction between obesity and the gut microbiota: relevance in nutrition. Annu. Rev. Nutr. 31, 15-31. doi: 10.1146/annurev-nutr-072610-145146

Dewulf, E. M., Cani, P. D., Claus, S. P., Fuentes, S., Puylaert, P. G. B., Neyrinck, A. M., et al. (2013). Insight into the prebiotic concept: lessons from an exploratory, double blind intervention study with inulin-type fructans in obese women. Gut 62, 1112-1121. doi: 10.1136/gutjnl-2012303304

DiBaise, J. K., Zhang, H., Crowell, M. D., Krajmalnik-Brown, R., Decker, G. A., and Rittmann, B. E. (2008). Gut microbiota and its possible relationship with obesity. Mayo Clin. Proc. 83, 460-469. doi: 10.4065/83.4.460

Du, F., Virtue, A., Wang, H., and Yang, X.-F. (2013). Metabolomic analyses for atherosclerosis, diabetes, and obesity. Biomark. Res. 1:17. doi: 10.1186/20507771-1-17 
Dumas, M.-E., Barton, R. H., Toye, A., Cloarec, O., Blancher, C., Rothwell, A., et al. (2006). Metabolic profiling reveals a contribution of gut microbiota to fatty liver phenotype in insulin-resistant mice. Proc. Natl. Acad. Sci. U.S.A. 103, 12511-12516. doi: 10.1073/pnas.0601056103

Duncan, S. H., Holtrop, G., Lobley, G. E., Calder, A. G., Stewart, C. S., and Flint, H. J. (2004). Contribution of acetate to butyrate formation by human faecal bacteria. Br. J. Nutr. 91, 915-923. doi: 10.1079/BJN20041150

Faber, J. H., Malmodin, D., Toft, H., Maher, A. D., Crockford, D., Holmes, E., et al. (2007). Metabonomics in diabetes research. J. Diabetes Sci. Technol. 1, 549-557. doi: $10.1177 / 193229680700100413$

Fukiya, S., Arata, M., Kawashima, H., Yoshida, D., Kaneko, M., Minamida, K., et al. (2009). Conversion of cholic acid and chenodeoxycholic acid into their 7-oxo derivatives by Bacteroides intestinalis AM-1 isolated from human feces. FEMS Microbiol. Lett. 293, 263-270. doi: 10.1111/j.1574-6968.2009.01531.x

Gibson, G. R., Scott, K. P., Rastall, R. A., Tuohy, K. M., Hotchkiss, A., DubertFerrandon, A., et al. (2010). Dietary prebiotics: current status and new definition. Food Sci. Technol. Bull. Funct. Foods 7, 1-19. doi: 10.1616/14762137.15880

Gipson, G. T., Tatsuoka, K. S., Ball, R. J., Sokhansanj, B. A., Hansen, M. K., Ryan, T. E., et al. (2008). Multi-platform investigation of the metabolome in a leptin receptor defective murine model of type 2 diabetes. Mol. Biosyst. 4, 1015-1023. doi: 10.1039/b807332e

Guan, M., Xie, L., Diao, C., Wang, N., Hu, W., Zheng, Y., et al. (2013). Systemic perturbations of key metabolites in diabetic rats during the evolution of diabetes studied by urine metabonomics. PLOS ONE 8:e60409. doi: 10.1371/journal.pone.0060409

Hadacek, F. (2015). Low-molecular-weight metabolite systems chemistry. Front. Environ. Sci. 3:12. doi: 10.3389/fenvs.2015.00012

Harris, K., Kassis, A., Major, G., and Chou, C. J. (2012). Is the gut microbiota a new factor contributing to obesity and its metabolic disorders? J. Obes. 2012:879151. doi: $10.1155 / 2012 / 879151$

Hofmann, A. F., and Hagey, L. R. (2008). Bile acids: chemistry, pathochemistry, biology, pathobiology, and therapeutics. Cell. Mol. Life Sci. 65, 2461-2483. doi: 10.1007/s00018-008-7568-6

Hosseini, E., Grootaert, C., Verstraete, W., and Van de Wiele, T. (2011). Propionate as a health-promoting microbial metabolite in the human gut. Nutr. Rev. 69, 245-258. doi: 10.1111/j.1753-4887.2011.00388.x

Hu, F. B., Manson, J. E., Stampfer, M. J., Colditz, G., Liu, S., Solomon, C. G., et al. (2001). Diet, lifestyle, and the risk of type 2 diabetes mellitus in women. N. Engl. J. Med. 345, 790-797. doi: 10.1056/NEJMoa010492

Huang, C.-F., Cheng, M.-L., Fan, C.-M., Hong, C.-Y., and Shiao, M.-S, (2012). Nicotinuric acid: a potential marker of metabolic syndrome through a metabolomics-based approach. Diabetes Care 36, 1729-1731. doi: $10.2337 / \mathrm{dc} 12-1067$

Huo, T., Cai, S., Lu, X., Sha, Y., Yu, M., and Li, F. (2009). Metabonomic study of biochemical changes in the serum of type 2 diabetes mellitus patients after the treatment of metformin hydrochloride. J. Pharm. Biomed. Anal. 49, 976-982. doi: 10.1016/j.jpba.2009.01.008

Huo, T., Xiong, Z., Lu, X., and Cai, S. (2015). Metabonomic study of biochemical changes in urinary of type 2 diabetes mellitus patients after the treatment of sulfonylurea antidiabetic drugs based on ultra-performance liquid chromatography/mass spectrometry. Biomed. Chromatogr. 29, 115-122. doi: 10.1002/bmc.3247

Jones, M. L., Martoni, C. J., Ganopolsky, J. G., Labbé, A., and Prakash, S. (2014). The human microbiome and bile acid metabolism: dysbiosis, dysmetabolism, disease and intervention. Exp. Opin. Biol. Ther. 14, 467-482. doi: $10.1517 / 14712598.2014 .880420$

Kawamata, Y., Fujii, R., Hosoya, M., Harada, M., Yoshida, H., Miwa, M., et al. (2003). A G protein-coupled receptor responsive to bile acids. J. Biol. Chem. 278, 9435-9440. doi: 10.1074/jbc.M209706200

Koeth, R. A., Wang, Z., Levison, B. S., Buffa, J. A., Org, E., Sheehy, B. T., et al. (2013). Intestinal microbiota metabolism of L-carnitine, a nutrient in red meat, promotes atherosclerosis. Nat. Med. 19, 576-585. doi: 10.1038/ nm.3145

Kootte, R. S., Vrieze, A., Holleman, F., Dallinga-Thie, G. M., Zoetendal, E. G., de Vos, W. M., et al. (2012). The therapeutic potential of manipulating gut microbiota in obesity and type 2 diabetes mellitus. Diabetes Obes. Metab. 14, 112-120. doi: 10.1111/j.1463-1326.2011.01483.x
Kurland, I. J., Accili, D., Burant, C., Fischer, S. M., Kahn, B. B., Newgard, C. B., et al. (2013). Application of combined omics platforms to accelerate biomedical discovery in diabesity. Ann. N. Y. Acad. Sci. 1287, 1-16. doi: 10.1111/nyas.12116

Labbé, A., Ganopolsky, J. G., Martoni, C. J., Prakash, S., and Jones, M. L. (2014). Bacterial bile metabolising gene abundance in crohn's, ulcerative colitis and type 2 diabetes metagenomes. PLOS ONE 9:e115175. doi: 10.1371/journal.pone.0115175

Larsen, N., Vogensen, F. K., van den Berg, F. W. J., Nielsen, D. S., Andreasen, A. S., Pedersen, B. K., et al. (2010). Gut microbiota in human adults with type 2 diabetes differs from non-diabetic adults. PLOS ONE 5:e9085. doi: 10.1371/journal.pone.0009085

Lefebvre, P., Cariou, B., Lien, F., Kuipers, F., and Staels, B. (2009). Role of bile acids and bile acid receptors in metabolic regulation. Physiol. Rev. 89, 147-191. doi: 10.1152/physrev.00010.2008

Lever, M., and Slow, S. (2010). The clinical significance of betaine, an osmolyte with a key role in methyl group metabolism. Clin. Biochem. 43, 732-744. doi: 10.1016/j.clinbiochem.2010.03.009

Ley, R. E., Bäckhed, F., Turnbaugh, P., Lozupone, C. A., Knight, R. D., and Gordon, J. I. (2005). Obesity alters gut microbial ecology. Proc. Natl. Acad. Sci. U.S.A. 102, 11070-11075. doi: 10.1073/pnas.0504978102

Ley, R. E., Turnbaugh, P. J., Klein, S., and Gordon, J. I. (2006). Microbial ecology: human gut microbes associated with obesity. Nature 444, 1022-1023. doi: $10.1038 / 4441022 \mathrm{a}$

Li, M., Wang, B., Zhang, M. M., Rantalainen, M., Wang, S., Zhou, H., et al. (2008). Symbiotic gut microbes modulate human metabolic phenotypes. Proc. Natl. Acad. Sci. U.S.A. 105, 2117-2122. doi: 10.1073/pnas.0712038105

Llorach, R., Garcia-Aloy, M., Tulipani, S., Vazquez-fresno, R., and Andreslacueva, C. (2012). Nutrimetabolomic strategies to develop new biomarkers of intake and health effects. J. Agric. Food Chem. 60, 8797-8808. doi: 10.1021/jf301142b

Messana, I., Forni, F., Ferrari, F., Rossi, C., Giardina, B., and Zuppi, C. (1998). Proton nuclear magnetic resonance spectral profiles of urine in type II diabetic patients. Clin. Chem. 44, 1529-1534.

Micha, R., Wallace, S. K., and Mozaffarian, D. (2010). Red and processed meat consumption and risk of incident coronary heart disease, stroke, and diabetes mellitus: a systematic review and meta-analysis. Circulation 121, 2271-2283. doi: 10.1161/CIRCULATIONAHA.109.924977

Midtvedt, T. (1974). Microbial bile acid. Am. J. Clin. Nutr. 27, 1341-1347.

Mokdad, A. H., Ford, E. S., Bowman, B. A., Dietz, W. H., Vinicor, F., Bales, V. S., et al. (2003). Prevalence of obesity, diabetes, and obesity-related health risk factors, 2001. JAMA 289, 76-79. doi: 10.1001/jama.289.1.76

Moreno-Indias, I., Cardona, F., Tinahones, F. J., and Queipo-Ortuño, M. I. (2014). Impact of the gut microbiota on the development of obesity and type 2 diabetes mellitus. Front. Microbiol. 5:190. doi: 10.3389/fmicb.2014. 00190

Moreno-Navarrete, J. M., Ortega, F., Serino, M., Luche, E., Waget, A., Pardo, G., et al. (2012). Circulating lipopolysaccharide-binding protein (LBP) as a marker of obesity-related insulin resistance. Int. J. Obes. (Lond.) 36, 1442-1449. doi: 10.1038/ijo.2011.256

Musso, G., Gambino, R., and Cassader, M. (2010). Obesity, diabetes, and gut microbiota: the hygiene hypothesis expanded? Diabetes Care 33, 2277-2284. doi: $10.2337 / \mathrm{dc} 10-0556$

Nguyen, A., and Bouscarel, B. (2008). Bile acids and signal transduction: role in glucose homeostasis. Cell. Signal. 20, 2180-2197. doi: 10.1016/j.cellsig.2008.06.014

Nicholson, J. K., Holmes, E., Kinross, J., Burcelin, R., Gibson, G., Jia, W., et al. (2012). Host-gut microbiota metabolic interactions. Science 336, 1262-1267. doi: $10.1126 /$ science. 1223813

Piche, T., des Varannes, S. B., Sacher-Huvelin, S., Holst, J. J., Cuber, J. C., and Galmiche, J. P. (2003). Colonic fermentation influences lower esophageal sphincter function in gastroesophageal reflux disease. Gastroenterology 124, 894-902. doi: 10.1053/gast.2003.50159

Poirier, P., Giles, T. D., Bray, G. A., Hong, Y., Stern, J. S., Pi-Sunyer, F. X., et al. (2006). Obesity and cardiovascular disease: pathophysiology, evaluation, and effect of weight loss: an update of the 1997 American Heart Association Scientific Statement on Obesity and Heart Disease from the Obesity Committee of the Council on Nutrition Physical Activity, and Metabolism. Circulation 113, 898-918. doi: 10.1161/CIRCULATIONAHA.106.171016 
Prawitt, J., Caron, S., and Staels, B. (2011). Bile acid metabolism and the pathogenesis of type 2 diabetes. Curr. Diab. Rep. 11, 160-166. doi: 10.1007/s11892-011-0187-x

Psychogios, N., Hau, D. D., Peng, J., Guo, A. C., Mandal, R., Bouatra, S., et al. (2011). The human serum metabolome. PLoS ONE 6:e16957. doi: 10.1371/journal.pone.0016957

Qin, J., Li, Y., Cai, Z., Li, S. S., Zhu, J., Zhang, F., et al. (2012). A metagenome-wide association study of gut microbiota in type 2 diabetes. Nature 490, 55-60. doi: 10.1038 /nature 11450

Ridlon, J. M., Kang, D.-J., and Hylemon, P. B. (2006). Bile salt biotransformations by human intestinal bacteria. J. Lipid Res. 47, 241-259. doi: 10.1194/jlr.R500013-JLR200

Romano, K. A., Vivas, E. I., Amador-Noguez, D., and Rey, F. E. (2015). Intestinal microbiota composition modulates choline bioavailability from diet and accumulation of the proatherogenic metabolite trimethylamine- $\mathrm{N}$-oxide. MBio 6:e02481. doi: 10.1128/mBio.02481-14

Salek, R. M., Maguire, M. L., Bentley, E., Rubtsov, D. V., Hough, T., Cheeseman, M., et al. (2007). A metabolomic comparison of urinary changes in type 2 diabetes in mouse, rat, and human. Physiol. Genomics 29, 99-108. doi: 10.1152/physiolgenomics.00194.2006

Samuel, B. S., Shaito, A., Motoike, T., Rey, F. E., Backhed, F., Manchester, J. K., et al. (2008). Effects of the gut microbiota on host adiposity are modulated by the short-chain fatty-acid binding G protein-coupled receptor, Gpr41. Proc. Natl. Acad. Sci. U.S.A. 105, 16767-16772. doi: 10.1073/pnas.0808567105

Sanz, Y., Rastmanesh, R., Agostoni, C., and Agostonic, C. (2013). Understanding the role of gut microbes and probiotics in obesity: how far are we? Pharmacol. Res. 69, 144-155. doi: 10.1016/j.phrs.2012.10.021

Scalbert, A., Brennan, L., Fiehn, O., Hankemeier, T., Kristal, B. S., van Ommen, B., et al. (2009). Mass-spectrometry-based metabolomics: limitations and recommendations for future progress with particular focus on nutrition research. Metabolomics 5, 435-458. doi: 10.1007/s11306-009-0168-0

Schwiertz, A., Taras, D., Schäfer, K., Beijer, S., Bos, N. A., Donus, C., et al. (2010). Microbiota and SCFA in lean and overweight healthy subjects. Obesity (Silver Spring) 18, 190-195. doi: 10.1038/oby.2009.167

Shen, J., Obin, M. S., and Zhao, L. (2013). The gut microbiota, obesity and insulin resistance. Mol. Aspects Med. 34, 39-58. doi: 10.1016/j.mam.2012.11.001

Shoaie, S., Ghaffari, P., Kovatcheva-Datchary, P., Mardinoglu, A., Sen, P., Pujos-Guillot, E., et al. (2015). Quantifying diet-induced metabolic changes of the human gut microbiome. Cell Metab. 22, 320-331. doi: 10.1016/j.cmet.2015.07.001

Suhre, K., Meisinger, C., Döring, A., Altmaier, E., Belcredi, P., Gieger, C., et al. (2010). Metabolic footprint of diabetes: a multiplatform metabolomics study in an epidemiological setting. PLOS ONE 5:e13953. doi: 10.1371/journal.pone.0013953

Sun, L., Yu, Z., Ye, X., Zou, S., Li, H., Yu, D., et al. (2010). A marker of endotoxemia is associated with obesity and related metabolic disorders in apparently healthy Chinese. Diabetes Care 33, 1925-1932. doi: 10.2337/dc10-0340

Swann, J. R., Want, E. J., Geier, F. M., Spagou, K., Wilson, I. D., Sidaway, J. E., et al. (2011). Systemic gut microbial modulation of bile acid metabolism in host tissue compartments. Proc. Natl. Acad. Sci. U.S.A. 108(Suppl.), 4523-4530. doi: 10.1073/pnas.1006734107

Thomas, C., Gioiello, A., Noriega, L., Strehle, A., Oury, J., Rizzo, G., et al. (2009). TGR5-mediated bile acid sensing controls glucose homeostasis. Cell Metab. 10, 167-177. doi: 10.1016/j.cmet.2009.08.001

Thomas, C., Pellicciari, R., Pruzanski, M., Auwerx, J., and Schoonjans, K. (2008). Targeting bile-acid signalling for metabolic diseases. Nat. Rev. Drug Discov. 7 , 678-693. doi: 10.1038/nrd2619

Tilg, H., Moschen, A. R., and Kaser, A. (2009). Obesity and the microbiota. Gastroenterology 136, 1476-1483. doi: 10.1053/j.gastro.2009.03.030
Tremaroli, V., and Bäckhed, F. (2012). Functional interactions between the gut microbiota and host metabolism. Nature 489, 242-249. doi: 10.1038 /nature 11552

Turnbaugh, P. J., and Gordon, J. I. (2009). The core gut microbiome, energy balance and obesity. J. Physiol. 587, 4153-4158. doi: 10.1113/jphysiol. 2009.174136

Venema, K. (2010). Role of gut microbiota in the control of energy and carbohydrate metabolism. Curr. Opin. Clin. Nutr. Metab. Care 13, 432-438. doi: 10.1097/MCO.0b013e32833a8b60

Vrieze, A., Holleman, F., Zoetendal, E. G., de Vos, W. M., Hoekstra, J. B. L., and Nieuwdorp, M. (2010). The environment within: how gut microbiota may influence metabolism and body composition. Diabetologia 53, 606-613. doi: 10.1007/s00125-010-1662-7

Vrieze, A., Out, C., Fuentes, S., Jonker, L., Reuling, I., Kootte, R. S., et al. (2014). Impact of oral vancomycin on gut microbiota, bile acid metabolism, and insulin sensitivity. J. Hepatol. 60, 824-831. doi: 10.1016/j.jhep.2013.11.034

Wang, Z., Klipfell, E., Bennett, B. J., Koeth, R., Levison, B. S., Dugar, B., et al. (2011). Gut flora metabolism of phosphatidylcholine promotes cardiovascular disease. Nature 472, 57-63. doi: 10.1038/nature09922

Warrier, M., Shih, D. M., Burrows, A. C., Ferguson, D., Gromovsky, A. D., Brown, A. L., et al. (2015). The TMAO-generating enzyme flavin monooxygenase 3 is a central regulator of cholesterol balance. Cell Rep. doi: 10.1016/j.celrep.2014.12.036 [Epub ahead of print].,

Watanabe, M., Houten, S. M., Mataki, C., Christoffolete, M. A., Kim, B. W., Sato, H., et al. (2006). Bile acids induce energy expenditure by promoting intracellular thyroid hormone activation. Nature 439, 484-489. doi: 10.1038 /nature 04330

Wei, L., Liao, P., Wu, H., Li, X., Pei, F., Li, W., et al. (2008). Toxicological effects of cinnabar in rats by NMR-based metabolic profiling of urine and serum. Toxicol. Appl. Pharmacol. 227, 417-429. doi: 10.1016/j.taap.2007.11.015

Won, E.-Y., Yoon, M.-K., Kim, S.-W., Jung, Y., Bae, H.-W., Lee, D., et al. (2013). Gender-specific metabolomic profiling of obesity in leptindeficient ob/ob mice by $1 \mathrm{H}$ NMR spectroscopy. PLOS ONE 8:e75998. doi: 10.1371/journal.pone.0075998

World Health Organization [WHO] (2013). Obesidad y Sobrepeso, 311. Available at: http://www.who.int/mediacentre/factsheets/fs311/en/ [Accessed April 2, 2014].

Zeisel, S. H. (2000). Choline: an essential nutrient for humans. Nutrition 16, 669-671. doi: 10.1016/S0899-9007(00)00349-X

Zhang, X., Wang, Y., Hao, F., Zhou, X., Han, X., Tang, H., et al. (2009). Human serum metabonomic analysis reveals progression axes for glucose intolerance and insulin resistance statuses. J. Proteome Res. 8, 5188-5195. doi: $10.1021 /$ pr900524z

Zhao, X., Fritsche, J., Wang, J., Chen, J., Rittig, K., Schmitt-Kopplin, P., et al. (2010). Metabonomic fingerprints of fasting plasma and spot urine reveal human pre-diabetic metabolic traits. Metabolomics 6, 362-374. doi: 10.1007/s11306010-0203-1

Conflict of Interest Statement: The authors declare that the research was conducted in the absence of any commercial or financial relationships that could be construed as a potential conflict of interest.

Copyright (C) 2015 Palau-Rodriguez, Tulipani, Queipo-Ortuño, Urpi-Sarda, Tinahones and Andres-Lacueva. This is an open-access article distributed under the terms of the Creative Commons Attribution License (CC BY). The use, distribution or reproduction in other forums is permitted, provided the original author (s) or licensor are credited and that the original publication in this journal is cited, in accordance with accepted academic practice. No use, distribution or reproduction is permitted which does not comply with these terms. 